\title{
La formación del contador público como fuente de capital humano
}

\author{
Becerra, Ligia* \\ García, Ligia** \\ Higuerey, Angel ${ }^{* * *}$ \\ Paredes, Rodolfo ${ }^{\star \star \star \star}$
}

\section{Resumen}

El capital intelectual es una concepción novedosa, tipificada como un activo intangible que agrega valor a la empresa; está formado por tres componentes: el capital organizacional, el humano y el relacional. El capital humano está relacionado con el desarrollo y los conocimientos del individuo, que permite diferenciar a un profesional de otro. El objetivo de este trabajo es analizar la formación del Contador Público egresado de la Universidad de Los Andes (ULA) de Trujillo, como fuente de capital humano para su desempeño profesional. Los datos se recolectaron aplicando un cuestionario a una muestra aleatoria de 47 de estos profesionales, empleando para el análisis y presentación de los resultados técnicas estadísticas. La investigación evidencia que la mayoría de los encuestados poseen un nivel de satisfacción de medio a bajo con su formación de pregrado, señalando como principales debilidades del Plan de Estudio las áreas tributaria y cambiaria, el manejo instrumental del inglés y el conocimiento de las normativas que rigen el sistema económico. La formación en algunas de estas áreas la han mejorado a través de otras actividades de capacitación. No obstante reconocen algunas fortalezas tales como: el aprendizaje y dominio de conceptos y Principios Contables de Aceptación General, las matemáticas y estadística, el manejo de la contabilidad computarizada y las habilidades para analizar e interpretar problemas contables. Se concluye que a pesar de las deficiencias, la realización de cursos de actualización, junto con las fortalezas en la formación de la carrera, ha contribuido a que el contador público egresado de la ULA de Trujillo constituya un capital humano con ventaja competitiva.

Palabras clave: Capital Intelectual, Ventajas Competitivas, Formación del contador público.

\section{Recibido: 04-12-03. Aceptado: 05-09-21}

* $\quad$ Profesora de la Universidad de los Andes (ULA), Núcleo Universitario Rafael Rangel (NURR), Economista, MS en Administración. E-mail: limabesa@cantv.net, Autora para la correspondencia.

** Profesora de la ULA. NURR, Economista, MS en Desarrollo Agrario.

E-mail: nathaliegar21@hotmail.com

*** Profesor de la ULA. NURR, Administrador, Esp. en Derecho Tributario. E-mail: anahigo@ula.ve

**** Licenciado en Contaduría Pública. Email: rodolfo_paredes@cantv.net 


\title{
The Formation of Public Accountants as a Source of Human Capital
}

\begin{abstract}
Intellectual capital is a new concept, typified as an intangible good that gives added value to a company, and it is made up of three components: organizational capital, human capital, and relational capital. Human capital is related with the development and knowledge of individuals which differentiates one professional from another. The objective of this paper is to analyze the formation of a public accountant who graduates from the Los Andes University in Trujillo, as a source of human capital in the labour market. The data was collected through a questionnaire in a random sample of 47 professionals, employing statistical methods in the analysis and presentation of the results. The results indicated that the majority of those surveyed had middle to low rates of satisfaction with their undergraduate formation, pointing to weaknesses in the program in the areas of taxation and currency exchange, instrumental knowledge of English, and knowledge of the norms that control the economic system. Formation in some of these areas was improved through additional alternative training. However, those sampled recognized comparative strengths in the areas of accounting principles, general mathematics and statistics, and in computerized accounting management, and analysis and interpretation of accounting problems. The conclusion is that even though there are deficiencies, refresher and up-date courses, together with the strong points of the curriculum, have contributed to producing a ULA graduate with competitive advantages in the labour market.
\end{abstract}

Key words: Intellectual capital, competitive advantages, public accountant formation.

\section{Introducción}

La generación de empleo, riqueza y bienestar de un país depende, en gran medida, de la competitividad de la gente y de sus empresas; de allí la importancia que estas últimas le presten a mejorarla para desenvolverse en mercados cada vez más abiertos y competitivos. Esta situación estará determinada, por un lado por la dotación de recursos y capacidades, susceptible de aprovechar las oportunidades de negocio presentes, y por otra parte, por la habilidad de las empresas para acumular y desarrollar nuevos recursos que les permita crear ventajas competitivas en el futuro.

En este sentido al hacer referencia al término competitividad, tal como lo señalan Hamel y Prahalad citados por Gar- cía et al. (1999:1) "es una industria en crecimiento: los presidentes y los primeros ministros prometen mejorarla, los legisladores la debaten, los economistas la miden y los directores de los periódicos la resaltan". La competitividad entre los diferentes países, empresas, organizaciones y profesiones les permitirá mantenerse o mejorar su situación en el mercado.

Dentro de estas diferencias competitivas se encuentra como factor principal, el capital intelectual, entendiendo éste como el conjunto de activos intangibles de una organización que, aún cuando no están reflejados en los estados contables tradicionales, generan valor o tienen el potencial de generarlo en el futuro; y que los estudiosos del tema: Edvinsson y Malone (1997); Siliceo et al 
(1999); Steward (1997); Brooking (1997) y Mantilla(2000) no se han puesto de acuerdo para definir exactamente los elementos que lo componen, pero en rasgos generales se puede decir que está conformado por los siguientes: Capital organizacional, humano y relacional.

Dentro del capital organizacional, la capacidad de innovación de la empresa, puede considerarse, como la principal fuente de ventajas competitivas sostenibles ya que va a hacer posible la acumulación del resto de intangibles. De esta forma, los aspectos relativos a la gestión y desarrollo de esta capacidad empresarial, estarán vinculados al fomento del aprendizaje tanto individual como colectivo dentro de la organización a través de una adecuada gestión del conocimiento y de su capital humano.

De allí, que las empresas ya empiezan a darse cuenta que una de las claves para el éxito se encuentra en el sistema de dirección y desarrollo del capital humano; es decir, de sus trabajadores. En un momento determinado se puede obtener un buen resultado con base en circunstancias como un avance técnico que aumente la eficiencia de las operaciones, la aplicación de productos maduros a la satisfacción de necesidades no atendidas de mercados en expansión, o la evolución de tipos de cambio favorable a las exportaciones. Pero detrás de esas oportunidades hay siempre personas capaces de aprovecharlas o, incluso, crearlas.

De lo anterior se puede inferir que es necesario dotar a la organización y a las personas de buen liderazgo, comunicación, motivación, visión estratégica y coordinación, todo ello en una cultura de colaboración, aprendizaje y sentido de responsabilidad social. Un componente importante de las organizaciones es el Contador Público, profesional que está en capacidad de prestar sus servicios en cuanto a la elaboración de información financiera necesaria para la toma de decisiones, elaborar sistemas de información contables financieros y realizar auditorías.

Esta carrera es ofrecida por varias universidades a nivel nacional. Una de ellas es la Universidad de Los Andes (ULA) - Núcleo Universitario Rafael Rangel (NURR) en Trujillo, está plasmada en un plan de diez (10) semestres, conformado por las siguientes áreas de conocimiento: Contable Financiera (constituye el eje central del currículo), de Administración, de Economía, de Matemáticas y Estadística, Legal, de Computación, Cibernética y de Idiomas; incluyendo además, como parte de la formación profesional, las pasantías técnicas y profesionales y la elaboración de un trabajo especial de grado.

Estas son las áreas que diferencian a los Contadores Públicos egresados de la ULA-NURR, y que conforman la base del capital humano que éstos aportarán a las organizaciones donde se desempeñen. De las premisas anteriores parte el interés de la presente investigación, la cual se ha planteado como objetivo general analizar al capital humano como ventaja competitiva del Contador Público egresado de esta casa de estudio.

Esta investigación se fundamenta en una análisis del concepto de capital intelectual y sus elementos constituyentes; destacando el capital humano como fuente de ventaja competitiva de las organizaciones innovadoras y haciendo hincapié en el papel de las universidades en 
el desarrollo del capital humano, sobre la base del plan de estudio de la carrera de Contaduría Pública de la ULA-NURR.

De acuerdo con lo anterior, la investigación se tipifica como descriptiva utilizando un diseño no experimental transversal o de campo, ya que no se manipuló las variables objeto de investigación; obteniendo los datos de la realidad en un solo momento. La población estuvo conformada por 150 egresados de la Licenciatura en Contaduría Pública de la ULA-NURR, de las diferentes cohortes hasta diciembre de 2003, que fue obtenida de la base de datos de la Oficina de Registros Estudiantes de la universidad; se consideró una muestra de 47 egresados, seleccionada a partir de la aplicación de una prueba piloto con un nivel de confianza del $95 \%$ y un error máximo admisible del 7,5\%. Para la obtención de los datos se utilizó la encuesta, utilizando como instrumento un cuestionario, conformado por 32 preguntas, en su mayoría cerradas y de opción de respuestas única (tanto dicotómicas como utilizando una escala de acuerdo-desacuerdo) y múltiple. La confiabilidad del instrumento fue medida por el coeficiente Alfa-Cronbach, con un valor de 0,8394 .

Los datos recolectados fueron verificados, codificados y analizados mediante estadística descriptiva, específicamente las referidas a la distribución de frecuencias y porcentajes; para lo cual se utilizó el programa estadístico computarizado SPSS, versión 11.

\section{El capital intelectual y sus elementos constituyentes}

A partir de la última década del siglo XX los líderes de las organizaciones innovadoras han comenzado a reconocer que sus activos contables tradicionales no constituyen la principal fuente de ventajas competitivas; por el contrario, han aceptado que ciertos activos intangibles como el talento, las competencias, las habilidades individuales, las relaciones de mercado y la capacidad de manejar eficientemente el flujo de competencias son los elementos que otorgan verdaderas fortalezas a las organizaciones. Tales factores conforman el capital intelectual de una organización empresarial.

En opinión de Siliceo et al. (1999), el término capital intelectual en su concepción original hacía referencia al círculo e inventario de conocimientos expresados en tres grandes dimensiones: a) la tecnológica (patentes, productos, procesos y servicios), b) la información (conocimiento del entorno de la organización, de los clientes, de los proveedores, de la competencia, entre otros) y, c) las habilidades humano - administrativas (procesos de comunicación, el trabajo en equipo, capacitación del personal, la creatividad e innovación, el liderazgo, entre otros).

Luego, la dinámica del desarrollo empresarial ha demostrado que existe una estrecha relación entre el capital intelectual de una organización y el incremento de su competitividad. Algunos autores como Siliceo et al (1999: 143) han planteado que: "... las empresas visionarias, excelentes y de clase mundial tienen como prioridad estratégica su propia construcción, fortalecimiento interno y la optimización de su capital intelectual, sólo de esta manera pueden responder a las necesidades del mercado y del cliente, y por ello, ser empresas altamente rentables". 
Por su parte, Edvinsson y Malone (1997:5) conceptualizan el capital intelectual mediante la utilización de la siguiente metáfora: "Una corporación es como un árbol. Hay una parte que es visible (las frutas) y una parte que está oculta (las raíces). Si solamente te preocupas por las frutas, el árbol puede morir. Para que el árbol crezca y continúe dando frutos, será necesario que las raíces estén sanas y nutridas. Esto es válido para las empresas: si sólo nos concentramos en los frutos (los resultados financieros) e ignoramos los valores escondidos, la compañía no subsistirá en el largo plazo".

Bajo la perspectiva de estos autores, el capital intelectual puede definirse como el conjunto de activos intangibles de una organización que, aún cuando no están reflejados en los estados contables tradicionales, generan valor o tienen el potencial de generarlo en el futuro. En este sentido, los conocimientos de las personas clave de la empresa, la satisfacción de los empleados, el know-how de la empresa, la satisfacción de los clientes, entre otros, son activos que explican buena parte de la valoración que el mercado concede a una organización y que, sin embargo, no son recogidos en el valor contable de la misma. De la medición de este tipo de capital nadie se había preocupado, sin embargo, en la década de los noventa, la compañía de Seguros y Asesoría Financiera Skandia ${ }^{1}$ comenzó a realizar esfuerzos por evaluar y medir su capital intelectual, a través de la presentación de informes en sus memorias anuales, donde se refleja el verdadero valor de la compañía y las variables que influyen en la generación de este valor.

Identificar y medir el capital intelectual, tiene como objetivo convertir en visible este activo que también genera valor a la organización. El peso del capital intelectual sobre el valor de mercado de una organización es creciente y por lo tanto los esfuerzos deben dirigirse a medirlo y a gestionarlo eficientemente.

En este orden de ideas, Mantilla (2000) afirma que el término capital intelectual presenta connotaciones complejas y a menudo es utilizado como sinónimo de propiedad intelectual, activos intelectuales y activos de conocimiento.

La propiedad intelectual es una modalidad de capital intelectual que otorga derechos de propiedad al conocimiento y a los bienes bajo la forma de patentes, marcas registradas y copyright; mientras que los activos intelectuales o activos de conocimiento, se pueden entender como recursos basados en conocimiento, a través de los cuales una organización podrá desarrollar una fuente de ventaja competitiva, contribuyendo así a incrementar los beneficios futuros de la misma, dentro de esta categoría se ubican la tecnología, los procesos administrativos y de consultoría, entre otros.

1 Mayor Compañía escandinava de Seguros y Servicios Financieros, la cual inició operaciones en1855 en Suecia; actualmente es un Grupo internacional cuyo radio de acción se ha extendido en Asia, Europa y América, incluyendo países latinoamericanos como Chile, México y Colombia. 
Desde esta perspectiva, cabe mencionar que una organización, para desarrollar su capital intelectual, dispone de dos fuentes fundamentales que contribuyen de manera determinante a la generación de conocimiento: la compra y las actividades de desarrollo interno.

La primera vía implica la adquisición de una patente, la contratación del personal o de los servicios de una compañía que haya capturado y generado el conocimiento demandado, para luego transferirlo al interior de la organización. Tal estrategia para la adquisición del conocimiento, presenta dos limitaciones fundamentales: a) la compañía antes de darle aplicabilidad a este factor productivo, no está completamente segura de que la innovación o el desarrollo tecnológico negociado es el que realmente necesita la organización para elevar la competitividad empresarial y mantenerse en el mercado; y b) para transferir el conocimiento adquirido entre los agentes que integran la organización, se requiere la creación de una estructura que facilite la realización de los procesos de difusión y transferencia del conocimiento.

La segunda vía exige la realización de actividades relacionadas con el adiestramiento y la investigación y desarrollo. Esta fuente de generación de conocimiento, presenta la limitación de que generalmente no existe una correlación directa entre el volumen de las inversiones realizadas y los resultados finales obtenidos; así pequeñas inversiones pueden conducir a la generación de gran cantidad de conocimiento, mientras que grandes inversiones pueden fracasar.

De otro lado, analizar el capital intelectual de una organización, exige consi- derar las variables que lo constituyen; algunos autores como Brooking (1997), Edvinsson y Malone (1997), Steward (1997) y el Instituto Universitario Euroforum Escorial (1998) entre otros, han agrupado los elementos constituyentes del capital intelectual en categorías diversas. Brooking (1997) expone que el capital intelectual de una empresa puede dividirse en cuatro categorías: a) activos de mercado (potencial derivado de los bienes inmateriales que guardan relación con el mercado), b) activos de propiedad intelectual (know-how, secretos de fabricación, copyright, patentes, derechos de diseño, marcas de fábrica y servicios), c) activos centrados en el individuo (cualidades que conforman al hombre y que hacen que sea lo que es) y d) activos de infraestructura (tecnologías, metodologías y procesos que hacen posible el funcionamiento de la organización).

Otra tipificación del capital intelectual es la expuesta por Edvinsson y Malone (1997), Steward (1997) y el Instituto Universitario Euroforum Escorial (1998), según los cuales este activo intangible está constituido por: a) Capital humano que comprende el conjunto de conocimientos tácitos y explícitos, habilidades, competencias y experiencias que el personal de una organización ha adquirido tanto en su formación como en su desempeño laboral, b) Capital estructural que incluye las capacidades organizacionales desarrolladas para satisfacer los requerimientos del mercado tales como patentes, marcas registradas, sistemas de tecnología de información, esfuerzos en investigación y desarrollo, entre otros y, c) Capital relacional, conformado a su vez por el capital clientela y el capital organi- 
zacional, este último se subdivide en capital innovación y capital proceso.

Por tanto, se puede expresar que el capital intelectual es una variable que sirve para medir el conocimiento organizativo y describir cómo son los recursos intangibles de una empresa. La esencia del capital intelectual es explicar la capacidad de futuras ganancias de la empresa con una perspectiva más profunda, amplia y humana que la presentada en los informes financieros.

El capital humano, como componente del capital intelectual, destaca como generador de ventajas competitivas en las organizaciones inteligentes; las cuales tienen una visión y misión que les permite ser sensibles para captar los cambios del entorno y prever las posibilidades de acción para insertarse en los competitivos mercados actuales.

\section{Capital humano y organizaciones innovadoras}

El capital humano se refiere al conocimiento -explícito o tácito- que poseen los trabajadores, útil para la empresa donde laboran, así como su capacidad para reproducirlo, es decir, su capacidad de aprender (Instituto Universitario Euroforum Escorial, 1998); es la base de la generación de los otros dos tipos que componen el capital intelectual. Una forma sencilla de distinguirlo del resto de los componentes del capital intelectual es que la empresa no lo posee y no lo puede comprar, sólo alquilarlo durante un periodo de tiempo; al decir de Altuve (2002) agrega valor a la empresa. En torno a esto se habla de los activos centrados en el individuo, los cuales están conformados por la pericia colectiva, la capacidad creativa, la habilidad para resolver problemas, el liderazgo y la capacidad empresarial y de gestión que poseen los trabajadores de toda organización. Según Steward (1997:25) el capital humano puede entenderse como: "Las capacidades individuales, el conocimiento, las habilidades y la experiencia de los empleados y directivos de la compañía. No puede ser adueñado por la organización. El Capital Humano puede estar en mayor o menor medida aferrado a la firma, según sean empleados incentivados a permanecer en la firma por programas de incentivos y capacidades de desarrollo personal".

En síntesis, a diferencia del capital tradicional de las empresas, el capital humano es propiedad del individuo y se caracteriza por ser renovable ya que debido a la obsolescencia del conocimiento, las organizaciones inteligentes han comenzado a crear el ambiente adecuado para que su personal aprenda constantemente y desarrolle la capacidad para regenerar el conocimiento, para así generar innovaciones tecnológicas y productivas que permitan a la empresa ser más eficiente y obtener ventajas competitivas.

En este sentido, cabe destacar que desde el mundo de las nuevas tendencias de la organización, se habla actualmente de las organizaciones que aprenden, para reflejar que lo importante es el aprendizaje, el no quedarse obsoleto y tener a toda la organización alerta ante las lecturas del mundo externo, las tendencias, las nuevas tecnologías, las nuevas realidades. Hoy una empresa no puede sobrevivir sin aprender; claro está, para ello es necesario crear una cultura de 
aprendizaje frente a una cultura del apoltronamiento o a una cultura del hacer.

Benavides (2002:71), expresa que la competitividad es la "capacidad de las empresas o industrias de competir y posicionarse en los mercados y los contextos de manera sostenible y a largo plazo". Esta definición concentra la clave con la cual se logra competir y además de ello, propicia la vía de establecerse de manera sostenible. Esto ajustado al recurso humano como capital humano, permite aseverar que la competitividad es la herramienta con que las personas, valiéndose de sus capacidades y cualidades, logran diferenciarse de los demás para así garantizar su permanencia y vigencia en los contextos y realidades.

De esta posición, se derivan algunas herramientas que integradas proveen el éxito competitivo; una de ellas es la competencia, que no es mas que los "comportamientos y destrezas visibles que la persona aporta en un empleo para cumplir con sus responsabilidades de manera eficaz y satisfactoria" (Benavides, 2002:72); es decir, son aquellos atributos observables en el individuo que lo caracterizan en el desempeño de sus actividades laborales.

En este contexto, las competencias son las unidades del conocimiento; son elementos que posee el individuo y son imprescindibles para desarrollar con éxito una actividad; son unidades relacionadas con su manera de ser, su manera de pensar y, finalmente, su manera de actuar. Aunque hay muchas definiciones de lo que son las competencias, se puede decir que son el conjunto de conocimientos, habilidades, cualidades y aptitudes que tienen las personas y que les predis- pone a realizar un conjunto de actividades con un buen nivel de desempeño.

Los individuos son fuentes de competitividad que deben convertirse en ventaja competitiva. El conocimiento de las personas y las capacidades de aprendizaje deben ser estimulados y regulados correctamente hacia un crecimiento efectivo, innovación constante y rentabilidad. En tal sentido, Ledesma (2001) manifiesta que la dirección de personas como ventaja competitiva sostenible hace que las empresas empiezan a darse cuenta de que una de las claves para el éxito se encuentra en el sistema de dirección de personal.

A nivel general, las bases para un sistema de dirección de capital humano que favorezca el éxito de la empresa son bien conocidas. Es necesario dotar a la organización y a las personas de buen liderazgo, comunicación, motivación, visión estratégica y coordinación. Todo ello en una cultura de colaboración, aprendizaje y sentido de responsabilidad social. A nivel concreto, todos estos conceptos son muy difíciles de implantar y desarrollar, y eso, en expresiones de Ledesma (2001) por tres razones a saber:

En primer lugar, cada uno de esos conceptos es ambiguo en sí mismo. Por un lado, por ejemplo, parece que todos pueden identificar con relativa facilidad situaciones de buen y mal liderazgo. Pero, por otro, resulta tremendamente complicado ponerse de acuerdo acerca de qué constituye la esencia del buen liderazgo. Que existan prácticamente tantas teorías sobre el liderazgo como autores que escriben sobre el tema, demuestra que se trata de fenómenos importantes pero difíciles de comprender. $Y$ esto 
acerca del liderazgo se aplica al resto de conceptos como motivación, comunicación, aprendizaje, entre otros en que se basa la dirección de personal.

En segundo lugar, la implantación y efectividad de cada uno de esos conceptos es muy sensible al tejido interno y externo de la empresa; y como no hay dos empresas iguales, no existe una receta universalmente válida sobre cómo, por ejemplo, diseñar un sistema de comunicación o cómo desarrollar la capacidad de liderazgo.

En tercer lugar, parece también claro que todos esos conceptos son interdependientes y tienen naturaleza sistémica, por ejemplo, difícilmente será efectiva una buena coordinación entre unidades organizativas sin un buen liderazgo en la organización, o una buena motivación del personal sin buenos sistemas de comunicación. Es decir, que no basta con hacer las cosas bien en una de esas facetas, sino que es necesario un mínimo de excelencia en todas o casi todas ellas. Por otro lado, en relación con su naturaleza sistémica, parece también claro que el efecto de cada uno de esos conceptos no es simplemente aditivo "dos más tres igual cinco", sino multiplicativo, "dos por tres igual seis". Y con ello se amplifica la ventaja de la empresa que logra un mínimo de excelencia en todos, o la mayoría de esos factores.

En este sentido, Porter (1985) establece una jerarquía de fuentes de ventaja competitiva, distinguiendo entre ventajas de orden inferior, tales como bajos costos de mano de obra o materias primas baratas, que son fáciles de imitar, y ventajas de orden superior, referidas fundamentalmente a la diferenciación. La mejora de comunicaciones y transportes, y la posibilidad de ubicar las plantas de producción en aquellos lugares donde se encuentren los insumos más baratos, provocan la difícil sostenibilidad de las ventajas en costos. Las ventajas de orden superior son más difíciles de imitar y, por tanto, más sostenibles, debido a que requieren técnicas y capacidades más avanzadas, tales como personal más especializado y con elevada formación (capital humano), capacidad técnica interna (capital tecnológico), inversiones acumuladas en marketing (capital comercial) y una adecuada cultura empresarial y estructura organizativa que faciliten el desarrollo de los anteriores aspectos, tanto en el interior de la empresa como a través de relaciones estrechas con proveedores y clientes (capital organizativo). Así, las ventajas de diferenciación suelen ser más duraderas debido a que los recursos en los que se apoyan son fundamentalmente intangibles. En definitiva, la evolución del entorno empresarial está elevando la importancia de los intangibles, ya que hay una creciente necesidad de ofrecer ventajas competitivas vinculadas a la diferenciación, las cuales se apoyan, como se ha expuesto, en este tipo de recursos.

\section{El papel de las universidades en el desarrollo del capital humano}

El capital humano se nutre con la educación; ésta comienza con los rudimentos elementales de la escuela primaria, pasando por las distintas etapas de la formación educacional escogida por la persona de acuerdo con la cantidad y ca- 
lidad de conocimientos que el individuo haya decidido incorporar; en esa misma medida se valorizará su capital humano.

Estos conocimientos pueden ser tácitos, explícitos e implícitos. Los primeros son aquellos que existen y pueden ser utilizados por los individuos, pero que resulta muy difícil de explicarlos de manera oral o escrita, haciéndose necesario que la organización conozca que los posee y se asegure de tratarlos como un valioso activo, para evitar que la competencia los adquiera. Los explícitos se pueden documentar por escrito, se hallan bien organizados en el cerebro del individuo pudiéndose escribir en forma de libros, manuales y procedimientos. Por último, los implícitos se encuentran ocultos en los procedimientos operativos, en los métodos e incluso en la cultura corporativa de la empresa y que son difíciles de identificar y aprender.

En el caso de los Contadores Públicos, estos conocimientos, que conforman su capital humano, se adquieren y desarrollan en los institutos de educación superior, marcando diferencia en la calidad de ellos, el pensum de la universidad de donde sean egresados.

La Universidad se presenta como una organización social, en la cual se forman individuos portadores de un conjunto de conocimientos que los califican para el ejercicio profesional y la vida en sociedad; se crean conocimientos mediante la investigación en diversas disciplinas científicas y se transfieren éstos a la sociedad, utilizables para resolver problemas de desarrollo.

Concretamente, la formación académica del Contador Público de la ULANURR está estructurada y plasmada en el plan de estudios de esta carrera, y define al Licenciado en Contaduría Pública como "... un profesional que está en capacidad de brindar servicios y elaborar sistemas de información contables financieros, en el ejercicio independiente de la profesión, o en los servicios prestados bajo la relación de dependencia" (Rodríguez et al; 1995: 8); es decir, un perfil bastante amplio que permite al egresado resolver problemas generales y básicos inherentes a su profesión.

La formación profesional está plasmada en este plan, a lo largo de los diez (10) semestres, integrado por las siguientes áreas de conocimiento: Contable Financiera (constituye el eje central del currículo), de Administración, de Economía, de Matemáticas y Estadística, Legal, de Computación, Cibernética y de Idiomas; incluyendo además de estas áreas, como parte de la formación profesional, las pasantías técnicas y profesionales y la elaboración de un trabajo especial de grado.

El Licenciado en Contaduría Pública es un profesional que puede ejercer en forma dependiente e independiente, con conocimientos amplios en las áreas contables financieras, con capacidad para analizar, interpretar, y tomar decisiones, de orden financiero económico, en cualquier tipo de organización. Este profesional desarrollará las habilidades de seleccionar, procesar, registrar, interpretar, tomar decisiones, elaborar y presentar informes, diseñar e implementar sistemas de información contable financiera. En este sentido, el Contador Público egresado de la ULA NURR, de acuerdo con el plan de estudios (Rodríguez et al., 1995: 15-16), deberá poseer las siguientes características: 1) Eficiencia en el desarrollo de sus funciones y 
tareas, 2) Desarrollo de su personalidad en relación a las funciones propias de su formación profesional, tales como: Conocimientos de los conceptos básicos en el área contable financiera; habilidades para comunicarse; ética profesional, responsabilidad y creatividad: aptitud de análisis e interpretación de resultados; elaborar sistemas de información contables; asistir o asesorar a particulares y empresas y actuar en calidad de auditor interno o externo (ejercicio libre profesional) y 3) Capacidad para desarrollar trabajos de investigación en el área contable financiera en cualquier empresa.

Estas son las características que diferencian a los Contadores Públicos egresados de la ULA-NURR, y que conforman la base del capital humano que estos profesionales aportan a las organizaciones y empresas donde se desempeñen.

\section{Resultados}

En relación con la situación laboral de los Contadores Públicos egresados de la ULA - NURR se encontró que en su totalidad forman parte de la oferta de trabajo; es decir, están incorporados a la población económicamente activa, contando con un potencial de capital humano susceptible de agregar valor a cualquier organización, pública o privada. Se detectó que el $57,4 \%$ de los encuestados, se encuentran actualmente laborando; mientras que los restantes no han podido ubicarse en un empleo que les permita desarrollar su potencial como profesionales. De los Contadores Públicos que están ocupados, un porcentaje importante $(92,6 \%)$ están laborando en su campo de trabajo y apenas un $7,4 \%$ se ubican en otro campo, desaprovechando sus conocimientos.

Los egresados de Contaduría Pública, pueden ejercer en cualquier sector de la economía, los egresados de la ULA - NURR están aproximadamente igual distribuidos en diversos campos, resaltando el sector público con un $36 \%$, el restante $64 \%$ se encuentra en el sector privado, bien sea laborando de manera dependiente en empresas, en firmas de contadores, o ejerciendo independiente; observándose que algunos de ellos laboran en varios campos simultáneamente.

Partiendo de que el capital humano es la combinación de conocimientos, habilidades, inventiva y capacidad de las personas para llevar acabo la tarea que tienen entre manos, podría expresarse que lo constituyen aquellas personas que generan riqueza porque saben como crear valor y producir contribución fructífera, debido a que tienen los conocimientos y las habilidades de implantar efectivamente soluciones creativas a los problemas.

La formulación anterior sustenta los resultados que se muestran en la Tabla 1, donde se aprecia el Nivel de Satisfacción con los conocimientos adquiridos por los Contadores Púlicos egresados de la ULA-NURR en sus estudios de pregrado; dichos conocimientos les permiten desarrollar sus competencias y abordar su desempeño profesional de tal manera que propicie su balance competitivo.

Este nivel de satisfacción en forma global, se puede considerar de medio a bajo, por cuanto un $56 \%$ de las opiniones de los encuestados, se ubican en un nivel Medianamente Satisfactorio o Deficiente; lo que indica la existencia de debilidades en el Plan de Estudio de la carrera, en 


\section{Tabla 1 \\ Contadores Públicos egresados de la ULA-NURR por Nivel de Satisfacción de los conocimientos adquiridos en sus estudios de pregrado}

\begin{tabular}{|c|c|c|c|c|c|c|c|c|c|c|}
\hline \multirow[t]{2}{*}{ Conocimientos } & \multicolumn{2}{|c|}{ AS } & \multicolumn{2}{|c|}{ MS } & \multicolumn{2}{|c|}{ MeS } & \multicolumn{2}{|c|}{$\mathrm{De}$} & \multicolumn{2}{|c|}{ Total } \\
\hline & $\mathrm{Fr}$ & $\%$ & $\mathrm{Fr}$ & $\%$ & $\mathrm{Fr}$ & $\%$ & $\mathrm{Fr}$ & $\%$ & $\mathrm{Fr}$ & $\%$ \\
\hline $\begin{array}{l}\text { Conceptos y principios de Con- } \\
\text { tabilidad General }\end{array}$ & 10 & 21,3 & 25 & 53,2 & 11 & 23,4 & 1 & 2,1 & 47 & 100,0 \\
\hline $\begin{array}{l}\text { Métodos y técnicas en el área } \\
\text { contable financiera }\end{array}$ & 10 & 21,3 & 18 & 38,3 & 19 & 40,4 & & 0,0 & 47 & 100,0 \\
\hline $\begin{array}{l}\text { Área administrativa como herra- } \\
\text { mienta para planificar, dirigir y } \\
\text { controlar }\end{array}$ & 6 & 12,8 & 20 & 42,6 & 20 & 42,6 & 1 & 2,1 & 47 & 100,0 \\
\hline $\begin{array}{l}\text { Sistema económico, cuentas } \\
\text { nacionales, inflación y análisis } \\
\text { macroeconómico }\end{array}$ & 7 & 14,9 & 13 & 27,7 & 22 & 46,8 & 5 & 10,6 & 47 & 100,0 \\
\hline $\begin{array}{l}\text { Matemática y estadística como } \\
\text { herramienta de apoyo operacio- } \\
\text { nal para desarrollar competen- } \\
\text { cias }\end{array}$ & 10 & 21,3 & 19 & 40,4 & 17 & 36,2 & 1 & 2,1 & 47 & 100,0 \\
\hline $\begin{array}{l}\text { Orientación relacionada con la } \\
\text { ética, moral y valores }\end{array}$ & 10 & 21,3 & 17 & 36,2 & 12 & 25,5 & 8 & 17,0 & 47 & 100,0 \\
\hline $\begin{array}{l}\text { Proceso de investigación orien- } \\
\text { tado a la solución de problemas }\end{array}$ & 6 & 12,8 & 10 & 21,3 & 24 & 51,1 & 7 & 14,9 & 47 & 100,0 \\
\hline $\begin{array}{l}\text { Leyes, normas y reglamentos } \\
\text { que rigen el sistema económico, } \\
\text { las relaciones ínter empresaria- } \\
\text { les y laborales }\end{array}$ & 1 & 2,1 & 12 & 25,5 & 29 & 61,7 & 5 & 10,6 & 47 & 100,0 \\
\hline $\begin{array}{l}\text { Ley del Ejercicio de la Contadu- } \\
\text { ría, los reglamentos y las nor- } \\
\text { mas establecidas por la FCCPV }\end{array}$ & 5 & 10,6 & 13 & 27,7 & 24 & 51,1 & 5 & 10,6 & 47 & 100,0 \\
\hline $\begin{array}{l}\text { Manejo de herramientas de } \\
\text { Contabilidad Computarizada }\end{array}$ & 8 & 17,0 & 21 & 44,7 & 17 & 36,2 & 1 & 2,1 & 47 & 100,0 \\
\hline Áreas tributaria y cambiaria & 3 & 6,4 & 2 & 4,3 & 28 & 59,6 & 14 & 29,8 & 47 & 100,0 \\
\hline $\begin{array}{l}\text { Diseño e implementación de sis- } \\
\text { temas de información contable }\end{array}$ & 2 & 4,3 & 14 & 29,8 & 26 & 55,3 & 5 & 10,6 & 47 & 100,0 \\
\hline Dominio instrumental del inglés & 3 & 6,4 & 4 & 8,5 & 27 & 57,4 & 13 & 27,7 & 47 & 100,0 \\
\hline
\end{tabular}

Fuente: Instrumento aplicado

Fr: Frecuencia AS: Altamente Satisfactorio; MS: Muy Satisfactorio; MeS: Medianamente Satisfactorio; De: Deficiente; ULA-NURR: Universidad de Los Andes (Venezuela) Núcleo Universitario Rafael Rangel. 
cuanto a conocimientos. Dentro de los aspectos sobre los cuales la opinión de los egresados se inclina hacia estas posiciones se encuentran: conocimientos en las áreas tributarias y cambiarias con un $89,4 \%$, en el dominio instrumental del inglés con un $85,1 \%$, de las leyes normas y reglamentos que rigen el sistema económico y las relaciones inter-empresariales y laborales con un $72,3 \%$, sobre el proceso de investigación orientado a la solución de problemas con un $66 \%$, en cuanto al diseño e implementación de sistemas de información contable con un $66 \%$; así mismo los aportes recibidos sobre la Ley del Ejercicio de la Contaduría, los reglamentos y las normas establecidas por la Federación de Colegios de Contadores Públicos de Venezuela (FCCPV) con un $61,7 \%$ y por último el manejo del sistema económico, cuentas nacionales, el fenómeno inflacionario y el análisis macroeconómico y sus efectos con un $57,4 \%$.

En contraposición, los resultados que muestran las áreas de conocimiento con las cuales los egresados se identifican como Altamente Satisfechos o Muy Satisfechos son: el aprendizaje, dominio de conceptos y principios contables de aceptación general (PCGA) con un $74,5 \%$; el área de matemáticas y estadísticas y la contabilidad computarizada como herramientas de apoyo operacional para desarrollar las competencias profesionales se soportan con un $61,7 \%$; el aprendizaje y dominio de métodos y técnicas en el área contable financiera con un $59,6 \%$; la orientación relacionada con la ética, moral y valores representada por un $57,4 \%$ y finalmente los conocimientos adquiridos en el área administrativa como herramientas para planificar, dirigir y con- trolar con un $55,4 \%$. Estos aspectos se constituyen en las principales fortalezas, que en la opinión de los mismos egresados son considerados variable clave del éxito para su desempeño profesional.

Resalta que dentro de las debilidades se encuentran: aspectos en materia tributaria, los sistemas de información contable y de idiomas (el inglés) que son claves para ser competitivos y desempeñarse en un mercado global, ya que éstos son indispensables para el desarrollo de una buena profesión. No se concibe, un contador público con debilidades en estas áreas. Sin embargo, existen aspectos de la formación del egresado de contaduría pública de esta institución como son la comprensión sobre las reglas generales y la aplicación de tecnología en el campo contable que le permiten desarrollarse $y$ competir en el mercado; aspectos primordiales para el buen desempeño de un contador público en las organizaciones modernas e indispensables para la toma de decisiones por parte de la gerencia de las empresas, así como para el mejor control de los organismos públicos.

Considerando como base el plan de estudios de la carrera de Contaduría Pública del ULA-NURR, el egresado de esta casa de estudio debe adquirir habilidades inherentes como son: seleccionar, procesar, registrar, interpretar, tomar decisiones, elaborar y presentar informes, diseñar e implementar sistemas de información contables financieros. La opinión de los encuestados acerca de las capacidades adquiridas sobre estas habilidades, se observa en la Tabla 2, y resalta que lo relacionado a las habilidades para su desempeño con respecto a la toma de decisiones y al diseño e implementación 


\section{Tabla 2}

\section{Capacidades adquiridas para el desempeño profesional por los egresados en Contaduría Pública de la ULA-NURR}

\begin{tabular}{|c|c|c|c|c|c|c|c|c|c|c|}
\hline \multirow[t]{2}{*}{ Capacidades } & \multicolumn{2}{|c|}{ AC } & \multicolumn{2}{|c|}{ MC } & \multicolumn{2}{|c|}{$\mathrm{MeC}$} & \multicolumn{2}{|c|}{ PC } & \multicolumn{2}{|c|}{ Total } \\
\hline & $\mathrm{Fr}$ & $\%$ & $\mathrm{Fr}$ & $\%$ & $\mathrm{Fr}$ & $\%$ & $\mathrm{Fr}$ & $\%$ & $\mathrm{Fr}$ & $\%$ \\
\hline $\begin{array}{l}\text { Analizar e interpretar problemas } \\
\text { contables }\end{array}$ & 5 & 10,6 & 24 & 51,1 & 18 & 38,3 & 0 & 0,0 & 47 & 100 \\
\hline $\begin{array}{l}\text { Toma de decisiones (económi- } \\
\text { cas y/o financieras) }\end{array}$ & 1 & 2,1 & 8 & 17,0 & 37 & 78,7 & 1 & 2,1 & 47 & 100 \\
\hline $\begin{array}{l}\text { Diseño e implementación de sis- } \\
\text { temas de información contable }\end{array}$ & 4 & 8,5 & 17 & 36,2 & 19 & 40,4 & 7 & 14,9 & 47 & 100 \\
\hline
\end{tabular}

Fuente: Instrumento aplicado.

Fr: Frecuencia; AC: Altamente Capacitado; MC: Muy Capacitado; MeC: Medianamente Capacitado; PC: Poco Capacitado; ULA-NURR: Universidad de Los Andes (Venezuela) Núcleo Universitario Rafael Rangel.

de sistemas de información contable son consideradas como no satisfactorias al opinar en un $80,8 \%$ y $55,3 \%$ respectivamente; es decir que las deficiencias en los conocimientos ya comentados se materializan en la adquisición de deficientes habilidades.

No obstante el análisis e interpretación de problemas contables, se convierte en una satisfacción con su capacitación al opinar en un $61,7 \%$ favorablemente a ella, siendo consistente con la fortaleza detectada en los conocimientos obtenidos por los egresados; de acuerdo a lo comentado en párrafos precedentes.

La contaduría Pública es una profesión que sufre cambios constantes a pesar de tener principios establecidos; por consiguiente, se requiere que el egresado esté en constante mejoramiento y actualización; además de esto, éste actúa en un campo muy competitivo donde solamente aquellos que resalten ocuparán las mejores posiciones o el mejor mercado.

En la Tabla 3 se muestra en síntesis los resultados recabados referentes a la realización de actividades de perfeccionamiento y/o mejoramiento profesional de los egresados de contaduría pública de la ULA-NURR, donde un $59,57 \%$ se han preocupado por perfeccionar y mejorar sus actitudes profesionales por medio de la realización de cursos; específicamente en las áreas de impuestos, legislación laboral, análisis e interpretación de Estados Financieros y los distintos software contable-administrativos indudablemente necesarios para el contador público de hoy; y que fueron detectados como debilidades de su formación.

De lo anterior se aprecia que el contador público egresado de la ULANURR, como un profesional responsable, aprovecha las oportunidades del entorno y realiza actividades de capacitación que le permitan minimizar las debilidades y potenciar las fortalezas; dentro de estas actividades se destacan: la materia tributaria y legal, así como los diferentes software de sistemas contables que se ofrecen en el mercado y que sirven de herramienta para su desempeño. 


\section{Tabla 3 \\ Actividades para el \\ perfeccionamiento, mejoramiento \\ y actualización profesional \\ realizadas por los egresados \\ en Contaduría Pública de la ULA-NURR}

\begin{tabular}{lcc}
\hline Actividad & Fr & $\%$ \\
\hline Especialización & 1 & 2,13 \\
Maestría & 1 & 2,13 \\
Cursos de Actualización & 28 & 59,57 \\
Ninguna & 17 & 36,17 \\
Totales & 47 & 100,00 \\
\hline
\end{tabular}

Fuente: Instrumento aplicado.

Fr: Frecuencia; ULA-NURR: Universidad de Los Andes (Venezuela) Núcleo Universitario Rafael Rangel.

Sin embargo, son pocos $(4,26 \%)$ los egresados de la ULA-NURR que están realizando estudios de Especialización y Maestría; aunque el campo de la Contaduría es altamente cambiante parece ser que estos profesionales no se interesan en profundizar sus conocimientos y actualizarse con un nivel superior para mantenerse en el mercado. Es decir, que la mayoría no le están dando la debida importancia a los estudios de cuarto nivel; estudios que en la actualidad son valiosos para las diferentes fuerzas empleadoras, ya que resalta una formación más sólida y especializada. Una razón que podría explicar esta situación, puede estar relacionada con los elevados costos de éstos sumado a que un porcentaje importante de los egresados $(42,6 \%)$ no se encontraban laborando cuando se tomó la muestra.

\section{Conclusión}

Del análisis de los resultados se evidencia que en la formación del Conta- dor Público egresado de la ULA-NURR se encuentran debilidades tanto en los conocimientos como en las habilidades adquiridas. Entre los primeros se encuentran el área tributaria, los sistemas de información contable y el manejo de ingles. Por otra parte dentro de las deficiencias en las habilidades se encuentra: La toma de decisiones y el diseño e implementación de sistemas de información contable. Aspectos que les dificulta ser competitivos en el mercado profesional, pues éstos son inherentes a la profesión.

Si se considera que el capital humano se refiere al conocimiento (explícito o tácito) que poseen los profesionales útil para una organización, se puede inferir que lo aportado por los Contadores Públicos egresados de la ULA-NURR, según su opinión, no es satisfactorio. En este sentido, estos profesionales concientes de sus debilidades, se preocupan por la realización de cursos y talleres relacionados con estos conocimientos. Esto es importante, porque el capital humano, también se considera como la capacidad para aprender y la mayoría de los egresados han realizados diferentes cursos.

No obstante, de acuerdo a la opinión de los encuestados, el capital humano aportado por los egresados en contaduría pública de la ULA-NURR, se ubica en las siguientes áreas de conocimientos que son consideradas como fortalezas: el aprendizaje, dominio de conceptos y principios contables de aceptación general (PCGA), el área de matemáticas y estadística y la contabilidad computarizada como herramientas de apoyo operacional, el aprendizaje y dominio de métodos y técnicas en el área contable financiera, la orientación relacionada con la ética, 
moral y valores y los conocimientos adquiridos en el área administrativa como herramientas para planificar, dirigir y controlar.

\section{Referencias Bibliográficas}

Altuve, José (2002), Capital Intelectual y Generación del Valor. Actualidad Contable. Año 5 № 5 . Venezuela. Universidad de Los Andes, Facultad de Ciencias Económicas y Sociales. Pp 23-29. Mérida, Venezuela.

Benavides, Olga (2002), Competencias y Competitividad. Diseño para Organizaciones Latinoamericanas. Bogotá D.C. McGraw-Hill.

Brooking, Annie (1997), El capital intelectual. Paidós. Barcelona, España.

Edvinsson, Leif y Malone, Michael S. (1997), EI Capital Intelectual. 1ra. Edición. Edición Norma. Bogotá.

INSTITUTO UNIVERSITARIO EUROFORUM ESCORIAL (1998), Conceptualización del Capital Intelectual. [Documento en línea] Disponible en: www.gestiondelconocimiento.com Consulta realizada el 25 de junio de 2002.

Flores M., Guillermo (2001), Capital intelectual en el ámbito de la contabilidad financiera. Revista Legis del Contador, No. 7, jul.-sep., pp. 79-115. Legis Editores S.A. Bogotá.
García L. Francisco; Mareo L., Bartolomé; Molina A., José Francisco; Quero Ramón, Diego (1999), La Capacidad de Innovación como Intangible Empresarial: Una Aproximación a través de la Gestión del Conocimiento. Espacios. Vol. 20 (3) 1999 [Revista en línea] Disponible en: www.revistaespacio.com

Ledesma, Luis (2001), Hacia una Dirección Estratégica del Capital Humano. [Documento en línea] Disponible en: www.lagente.com/cgibim/contenido. $\mathrm{pl}$ ?Art=50 Consulta realizada el $20 \mathrm{de}$ junio de [2002.

Mantilla, Samuel (2000), Capital Intelectual y Contabilidad del Conocimiento. ECOE Ediciones. Bogotá.

Porter, Michael (1985), La Ventaja Competitiva. New York. Free Press. USA.

Rodríguez, Ligia, Paredes, Fabricio, Zambrano Gustavo, Montilla, Tulio y García, Omaira (1995), Proyecto Académico de Licenciatura en Contaduría Pública de la Universidad de los Andes, Extensión Núcleo Universitario Rafael Rangel. Trujillo, Venezuela.

Siliceo, Alfonso, Casares David y Gonzalez José Luis (1999), Liderazgo, Valores y Cultura Organizacional. Hacia una organización competitiva. Editorial Mc Graw Hill. México.

Steward, Tom (1997), La nueva riqueza de las Organizaciones: El Capital Intelectual. Segunda Edición. GRANICA. 